\title{
Beam emittance calculation in the presence of an axially symmetric magnetic field
}

\author{
S. Nagaitsev and A. Shemyakin \\ Fermi National Accelerator Laboratory \\ P.O. Box 500, Batavia, IL 60510
}

\section{Introduction}

The Fermilab electron cooling project requires low transverse electron velocities (both coherent and incoherent) in the solenoidal cooling section [1]. One of the possible reasons for an increase of these velocities is aberrations introduced by optical elements in the axially symmetric portion of the beam channel (first of all, in the acceleration tube) preceding the cooling section. Most of these optical elements can be treated as thin lenses so that the aberrations can be characterized by an increase of an effective beam emittance as a function of the beam radius in this element. The goal of this note is to formulate a method that would allow to correctly estimate these aberrations in the presence of linear coupling and a non-zero longitudinal magnetic field at the cathode. This is of particular interest for electron beam simulation programs that are employed to optimize beam transport in the presence of a varying longitudinal magnetic field.

\section{One dimensional motion}

In the case of an uncoupled transverse particle motion, the beam phase space area in each plane can be characterized by an rms emittance, calculated as follows [2]:

$$
\begin{gathered}
\bar{\varepsilon}_{x}=\sqrt{\operatorname{det} \Sigma}=\sqrt{\left\langle(x-<x>)^{2}>\cdot<\left(x^{\prime}-<x^{\prime}>\right)^{2}>-<(x-<x>) \cdot\left(x^{\prime}-<x^{\prime}>\right)\right\rangle^{2}}, \\
\Sigma=\left(\begin{array}{cc}
<(x-<x>)^{2}> & <(x-<x>) \cdot\left(x^{\prime}-<x^{\prime}>\right)> \\
<(x-<x>) \cdot\left(x^{\prime}-<x^{\prime}>\right)> & <\left(x^{\prime}-<x^{\prime}>\right)^{2}>
\end{array}\right),
\end{gathered}
$$

where $x$ is a particle coordinate and $x^{\prime}$ is its angle, proportional to the particle's kinematic momentum. In a beam transport simulation, the operation noted by <> implies averaging over all $\mathrm{N}$ macroparticles:

$$
<>=\frac{1}{I} \sum_{i=1}^{N} f_{i} \cdot I_{i}
$$

where $I=\sum_{i=1}^{N} I_{i}$ is the total current. The following matrix equation

$$
X^{t} \Sigma^{-1} X=1
$$

where $X=\left[\begin{array}{c}x \\ x^{\prime}\end{array}\right]$, determines a two-dimensional beam phase-space ellipse. 


\section{Case of axial symmetry}

A direct application of Eq. (1) to the case of an axial symmetry gives the following result after averaging over the azimuth $\theta$ (macroparticles in most axially symmetric simulations are represented by rings):

$$
\begin{gathered}
x=r \cdot \cos \theta, \\
x^{\prime}=r^{\prime} \cdot \cos \theta-r \theta^{\prime} \cdot \sin \theta, \\
<x>=0, \\
<x^{\prime}>=0, \\
<x^{2}>=<\frac{1}{2 \pi} \int_{0}^{2 \pi}(r \cdot \cos \theta)^{2} \cdot d \theta>=\frac{<r^{2}>}{2}, \\
<x x^{\prime}>=<\frac{1}{2 \pi} \int_{0}^{2 \pi}\left(r^{\prime} \cdot \cos \theta-r \theta^{\prime} \cdot \sin \theta\right)^{2} \cdot d \theta>=\frac{<r^{\prime 2}+\left(r \theta^{\prime}\right)^{2}>}{2} \\
\end{gathered}
$$

and, after substitution of (4) into (1),

$$
\bar{\varepsilon}_{x}=\frac{1}{2} \cdot \sqrt{\left\langle r^{2}>\cdot<r^{\prime 2}+\left(r \theta^{\prime}\right)^{2}>-<r \cdot r^{\prime}\right\rangle^{2}} .
$$

The emittance calculated by Eq. (5) in the case of a non-zero longitudinal magnetic field at the cathode can be non-zero even with perfectly linear optical elements. For example, a homogenous round cold beam of electrons emitted along the magnetic field lines, accelerated, and then extracted from the field without aberrations, would have an emittance according to Eq. (5), which is proportional to the magnetic flux $\Phi=B R^{2}$ at the cathode:

$$
\bar{\varepsilon}_{x}=\frac{R^{2} \cdot e B}{8 \cdot p c}=\frac{e \Phi}{8 p c}
$$

where $p=\gamma \beta m c$ is the electron kinematic momentum, $R$ is the cathode radius and $B$ is the magnetic field at the cathode. The $\bar{\varepsilon}_{x}$ value is constant in a space free of the magnetic field and enters the beam envelope equation [3] in a manner identical to that of a statistical emittance ${ }^{1}$.

On the other hand, the emittance defined by Eq. (5) changes in varying longitudinal magnetic fields and can hardly be used to characterize the influence of aberrations.

\section{4D emittance}

We propose to use for an emittance calculation the value of a total volume of a 4-D phase-space ellipsoid. In analogy with (1),

\footnotetext{
${ }^{1}$ Effective emittance, often used in the envelope equation in place of the rms emittance, is defined being four times greater than the emittance in Eq. (5).
} 


$$
\begin{aligned}
& \bar{\varepsilon}_{4}=\sqrt{\operatorname{det} \Sigma_{4}} ;
\end{aligned}
$$

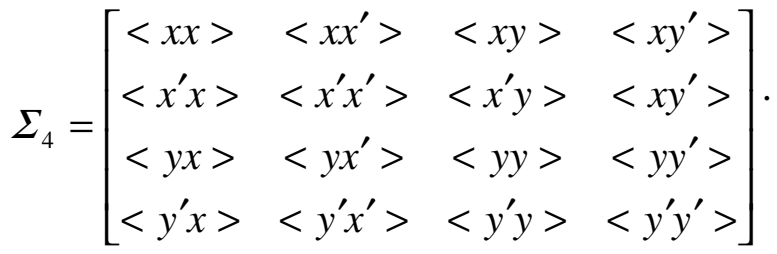

The matrix elements are calculated in analogy with (4):

$$
\begin{gathered}
<y^{2}>=<x^{2}>=\frac{<r^{2}>}{2} \\
<y^{\prime 2}>=<x^{\prime 2}>=\frac{<r^{\prime 2}+\left(r \theta^{\prime}\right)^{2}>}{2} \\
<y y^{\prime}>=<x x^{\prime}>=\frac{<r r^{\prime}>}{2} \\
<x y^{\prime}>=-<x^{\prime} y>=\frac{<r^{2} \theta^{\prime}>}{2} \\
<x^{\prime} y^{\prime}>=0
\end{gathered}
$$

After substitution (4) and (8) into (7), the final result is

$$
\begin{aligned}
& \operatorname{det} \Sigma_{4}=\operatorname{det}\left[\begin{array}{cccc}
\frac{<r^{2}>}{2} & \frac{\left.<r r^{\prime}\right\rangle}{2} & 0 & \frac{<r^{2} \theta^{\prime}>}{2} \\
\frac{<r r^{\prime}>}{2} & \frac{<r^{\prime 2}+\left(r \theta^{\prime}\right)^{2}>}{2} & -\frac{<r^{2} \theta^{\prime}>}{2} & 0 \\
0 & -\frac{<r^{2} \theta^{\prime}>}{2} & \frac{<r^{2}>}{2} & \frac{<r r^{\prime}>}{2} \\
\frac{<r^{2} \theta^{\prime}>}{2} & 0 & \frac{<r r^{\prime}>}{2} & \frac{<r^{\prime 2}+\left(r \theta^{\prime}\right)^{2}>}{2}
\end{array}\right]= \\
& =\frac{1}{16}\left(<r^{2}><r^{\prime 2}+\left(r \theta^{\prime}\right)^{2}>-<\left(r \cdot r^{\prime}\right)>^{2}-<r^{2} \theta^{\prime}>^{2}\right)^{2}
\end{aligned}
$$

For convenience, this $4 \mathrm{D}$ emittance can be presented in 2D units:

$$
\bar{\varepsilon}_{2}=\sqrt{\bar{\varepsilon}_{4}}=\frac{1}{2} \sqrt{<r^{2}><r^{\prime 2}+\left(r \theta^{\prime}\right)^{2}>-<r \cdot r^{\prime}>^{2}-<r^{2} \theta^{\prime}>^{2}} .
$$

Equation (10) thus represents a value of an rms beam emittance in an axially symmetric case. It does not contain the magnetic field explicitly and can be easily computed at any 
point along the beam trajectory. It is worth noting, where $\theta^{\prime}=$ const (rigid beam rotation) Eq. (10) gives the value of the emittance, which does not depend on $\theta^{\prime}$.

It has been shown elsewhere (see, for example, [4]) that the total phase space volume of a particle beam calculated with kinematic momenta is identical to the volume calculated with canonical momenta. This follows from Liouville's theorem. It also can be demonstrated that the determinant of a $\Sigma_{4}$ matrix, with the matrix elements containing canonical momenta instead of $x^{\prime}$, is identical to Eq. (9), at least in the case of a uniform magnetic field.

\section{Discussion}

It is of practical interest for the Fermilab electron cooling project to obtain an estimate for the minimum achievable rms values of transverse beam angles in a solenoidal cooling section [5] from Eq. (10). First, we would like to point out that all offdiagonal elements in the matrix $\Sigma_{4}$ of Eq. (9) are nonzero only for a coherent beam motion, i.e. convergence or rotation. We claim, however, that by (1) choosing an appropriate value of the uniform longitudinal magnetic field $B_{o}$ as well as by (2) adjusting the beam convergence angle at the entrance in such a field region, one can zero all these non-diagonal matrix elements. The first of these two conditions follows from the fact that the injection of a beam into the uniform field is equivalent to a rigid beam rotation or, alternately, to a substitution of $\theta^{\prime}$ with $\theta^{\prime}-e B_{\delta} /(2 p c)$. Such a substitution does not change the value of the matrix determinant. Thus, one can always choose the field value $B_{o}$ such that

$$
\frac{<r^{2}\left(\theta^{\prime}-e B_{o} / 2 p c\right)>}{2}=0 .
$$

The second of the above two conditions implies that the beam needs to be parallel (on average) as it enters the section with a uniform longitudinal magnetic field. Since the uniform magnetic field conserves the total transverse momentum, these two conditions guaranty that the $\Sigma_{4}$ matrix has only diagonal elements along the full length of a uniform field section. This specific case of a round parallel electron beam propagating in a solenoidal cooling section is, in fact, a final goal of the Electron Cooling R\&D effort. The estimate for the minimum achievable rms transverse beam angles can be written as follows:

$$
\sqrt{\left\langle\alpha_{\min }^{2}\right\rangle}=\frac{2 \cdot \bar{\varepsilon}_{2}}{\sqrt{\left.<r^{2}\right\rangle}} .
$$

\section{Acknowledgment}

Authors acknowledge useful discussions with A.Burov, D.Myakishev, A.Novokhatsky, and M.Tiunov.

\section{References}

[1] "Prospectus for an Electron Cooling System for the Recycler", Fermilab preprint FERMILAB-TM-2061, edited by J.A. MacLachlan, October 1998. 
[2] P.M. Lapostolle, IEEE Trans. Nucl. Sci. NS-18, 1101 (1971).

[3] J.D.Lawson, The Physics of Charged-Particle Beams, 1977.

[4] M. Reiser, Theory and Design of Charged Particle Beams, John Wiley \& Sons, Inc., 1994.

[5] S. Nagaitsev, A. Shemyakin, and V. Vostrikov, "Fermilab Electron Cooling Project: Estimates for the Cooling Section Solenoid", Fermilab preprint FERMILAB-FN-689, April 2000 (available at http://fnalpubs.fnal.gov ). 